\title{
Standardized multimodal intervention for stress-induced exhaustion disorder: an open trial in a clinical setting
}

\author{
Jakob Clason van de Leur ${ }^{1 *}$ (D, Monica Buhrman ${ }^{1}$, Fredrik Åhs², Alexander Rozental ${ }^{3}$ and Gunilla Brodda Jansen ${ }^{4}$
}

\begin{abstract}
Background: Long-term sick-leave due to stress-related ill-health is increasing in several economically developed countries. Even though different forms of interventions are administered in regular care for stress-related disorders, such as Stress-induced Exhaustion disorder (SED), the scientific evidence for the effectiveness of such treatments is sparse. The objective of this study was to explore changes in SED-symptoms and return-to-work-rates in a large group of SED-patients participating in a standardized Multimodal intervention (MMI) in a clinical setting.

Method: This open clinical trial tracked 390 patients who fulfilled the criteria for SED undergoing a 24-week MMI, including return-to-work-strategies. Before inclusion, all patients underwent a multi-professional assessment by a team of licensed physicians, licensed psychologists, and licensed physiotherapists. Self-rated questionnaires were administered before treatment, at treatment-start, mid-treatment, post-treatment, and at 12-month follow-up. Within-group change was evaluated over time with mixed-effects models. Beyond different symptoms, working time, sick-leave compensation, and adverse effects were also measured.
\end{abstract}

Results: There were significant improvements in symptoms of SED, burnout, anxiety, depression, and insomnia, with large within-group effect sizes $(d=0.91-1.76)$, improvements that were maintained at 12-month follow-up. Furthermore, there was a significant increase in quality of life and large improvements in average working time and sick-leave compensation. Some adverse effects were reported, mainly concerning an increase in stress, anxiety, and worry.

Conclusion: SED-patients participating in this standardized MMI reported large symptom alleviation, increased working time and reduced sick-leave compensation, indicating a beneficial treatment. There were some adverse effects, but no more so than other psychological treatments. This study confirms previous findings that high levels of depression and anxiety decrease to sub-clinical levels during treatment, while symptoms of SED also decline, yet still persists above sub-clinical levels at 12-month follow-up. On the whole, this open clinical trial suggests that a standardized MMI, administered in a clinical setting, improves symptoms and return-to-work rates in a clinically representative SED-population.

Trial registration: This study was registered on Clinicaltrials.gov 2017.12.02 (Identifier: NCT03360136).

Keywords: Stress-induced exhaustion disorder, Burnout, Long-term stress, Rehabilitation, Multimodal intervention, Negative effects

\footnotetext{
* Correspondence: jakob.clason-vandeleur@psyk.uu.se

'Department of Psychology, Uppsala University, Von Kraemers allé 1A, SE-752 37 Uppsala, Sweden

Full list of author information is available at the end of the article
}

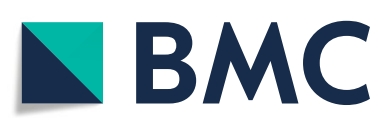

(c) The Author(s). 2021 Open Access This article is licensed under a Creative Commons Attribution 4.0 International License, which permits use, sharing, adaptation, distribution and reproduction in any medium or format, as long as you give appropriate credit to the original author(s) and the source, provide a link to the Creative Commons licence, and indicate if changes were made. The images or other third party material in this article are included in the article's Creative Commons licence, unless indicated otherwise in a credit line to the material. If material is not included in the article's Creative Commons licence and your intended use is not permitted by statutory regulation or exceeds the permitted use, you will need to obtain permission directly from the copyright holder. To view a copy of this licence, visit http://creativecommons.org/licenses/by/4.0/. The Creative Commons Public Domain Dedication waiver (http://creativecommons.org/publicdomain/zero/1.0/) applies to the data made available in this article, unless otherwise stated in a credit line to the data. 


\section{Background}

During the last two decades, long-term sick-leave due to stress-related ill-health has been increasing in several economically developed countries [1-4]. Long-term exposure to non-traumatic stressors (such as deficiencies in the work environment, high work-load, divorce, socioeconomic difficulties, and interpersonal conflicts) without sufficient recovery can lead to a debilitating state of exhaustion characterized by physical fatigue, cognitive impairments and sleep disturbances $[5,6]$.

In the field of work-psychology, the term burnout is often used to describe the end-stage of a long-term stress process, where exhaustion is a cardinal symptom $[7,8]$. The concept of burnout is, however, somewhat debated, and it has yet to be incorporated into any of the major diagnostic systems such as the Diagnostic and Statistical Manual of Mental Disorders (DSM-5) and the International Classification of Diseases, 10th edition (ICD-10 [9-12];). Because of this, as there is presently no internationally established terminology for stressinduced exhaustion, and different terms are used across different countries and disciplines, such as clinical burnout, job stress-related depression, exhaustion syndrome and neurasthenia $[5,13]$.

In Sweden, the diagnosis of "Stress-induced Exhaustion disorder" (SED) has been suggested to improve the identification and treatment of exhaustion due to longterm nontraumatic stress. SED (F43.8A) was accepted in the Swedish version of the ICD-10 in 2005 and can be regarded as an operationalization of severe "clinical burnout" [5]. Few studies have been published regarding the prevalence of SED, but in a recent cross-sectional study in northern Sweden, 4.2\% of 3406 participants reported a physician-based diagnosis of SED [14]. Furthermore, there is a growing body of literature indicating that SED could be associated with structural brain changes and biochemical irregularities [15-17]. Even though the diagnosis of SED as of now is utilized primarily in Sweden, there have been several international publications that suggest that SED is not in any way a unique Swedish condition [4, 18-23].

The main symptoms of SED are severe physical and mental exhaustion, following a period of at least 6 months of stress-related exposure. This is accompanied by an increased need for recovery after mental efforts, cognitive impairments such as short-term memory loss, concentration difficulties, and sound- and light sensitivity. Other symptoms such as sleep disturbances, dizziness, nausea, headaches, gastrointestinal problems, and longstanding pain in the neck and shoulders are common $[24,25]$.

Multimodal interventions (MMI), where several interventions are administered simultaneously by a team of different professionals working together, was developed for the rehabilitation of longstanding pain [26]. More recently, MMI has been recommended for the treatment of SED. MMI should, according to clinical recommendations, include lifestyle changes concerning the balance between activation and recuperation, some relaxation techniques, psychotherapy (preferably in a group context), and specific return-to-work-interventions that include communication with the patient's workplace. However, the empirical support for MMI in the treatment of SED is scarce [13].

One previous study has tracked the symptom development of SED-patients participating in MMR [24]. In this study, symptoms of depression and anxiety decreased rapidly within 3 months of treatment start, while symptoms of exhaustion declined more slowly, persisting to some extent 18 months later.

Two studies have investigated the effectiveness of MMI for SED [27, 28]. Both studies show within-group improvements over time in burnout symptoms and return-to-work in all groups, but few clinically significant differences are found between different treatments. It is also worth mentioning that existing studies of MMI for SED are seldom standardized, nor do they follow all of the clinical recommendations, making it harder to implement these treatments in clinical practice. In our understanding, previous studies of MMI for SED do not highlight or describe return-to-work-strategies as an integral core component of the rehabilitation process [24, 27,28 . A few studies of unimodal interventions specifically targeting return-to-work in SED-patients have shown that the effects on return-to-work are very small or negligible, compared to regular Cognitive Behavioral Therapy (CBT), Acceptance and Commitment Therapy, or no treatment at all [29-32].

To sum up, even though MMI is recommended for SED, the current body of research speaks little as to whether it is effective or not, and how the contents of an MMI should be structured to facilitate symptom improvement and return-to-work.

Another potential shortcoming of previous research is the lack of questionnaires, specifically measuring the symptoms of SED. Since the diagnosis of SED is in its early stages, researchers have had to turn to measures of burnout. Most often, the Shirom-Melamed Burnout Questionaire (SMBQ) has been used. However, burnout and SED may not be interchangeable constructs. The SMBQ consists of four sub-scales (physical fatigue, cognitive weariness, tension, and listlessness) while SED emphasizes symptoms of exhaustion and includes some dimensions (recovery, memory, hypersensitivity to sensory impressions, the experience of demands) not necessarily captured by the SMBQ. As an example of this, Saboonchi, Perski, and Grossi [33] found that the variance in SED could not be explained by the concept of 
burnout assessed with the SMBQ in a population of SED. In 2014 the Karolinska Exhaustion Disorder Scale (KEDS) was developed explicitly measuring the construct of SED [34]. As of today, KEDS has not yet been evaluated in any clinical trial with a SEDpopulation, even though the need for measuring the long-term symptoms of SED has frequently been emphasized $[24,35,36]$.

When evaluating a treatment program such as MMI for SED, which is already being implemented in regular care despite the lack of adequate evidence, it is crucial to measure adverse effects. Even though many evidencebased psychological treatments show an overall good effect, only about half of the patients respond to treatment, and some even deteriorate [37, 38]. Despite a marked increase in research of psychological interventions during the past two decades, the adverse effects from these treatments have been widely neglected, and the need for more rigorous exploration is frequently advocated $[39,40]$.

Therefore, this open clinical trial had three aims: 1) To determine whether SED-patients participating in a standardized MMI report symptom alleviation through self-rating questionnaires measuring SED, burnout, insomnia, anxiety, depression, and quality of life; 2) to determine whether SED-patients participating in a standardized MMI report an increase in return-to-workrates and 3) to evaluate the adverse effects of MMI for SED.

\section{Method}

\section{Study design}

This open clinical trial with a 12-month follow-up was part of a more extensive data collection from a standardized 24-week MMI for SED at two health care centers (PBM Sweden AB) in Stockholm, Sweden. These centers specialized in the rehabilitation of longstanding pain and SED and were part of a specialized health-care initiative called "The health care choice for treatment of longstanding pain with or without comorbidity, and Stressinduced Exhaustion disorder," on behalf of Health Care Services Stockholm County. The clinics received referrals from general practitioners, primary health care centers, and occupational health services from all over Stockholm. This study was registered on Clinicaltrials.gov (Identifier: NCT03360136), approved by the Regional Ethical Review Board in Stockholm, Sweden (Approval Nr. 2016/1834-31/2) and followed the ethical principles of the Declaration of Helsinki.

\section{Participants and recruitment procedure}

After being referred, the patients went through a multiprofessional team assessment performed by a licensed physician (45 $\mathrm{min})$, a licensed psychologist $(60 \mathrm{~min})$ and a licensed physiotherapist ( $45 \mathrm{~min}$ ), together with a survey of baseline characteristics and several self-rating questionnaires (see measurements), after which a $30 \mathrm{~min}$ summarized assessment was returned to the patient by the team. A total number of 662 patients underwent a multi-professional assessment between September 2017 and April 2018. Out of these, 173 did not fulfill the inclusion criteria for participation in the MMI, two patients were included in the rehabilitation program for longstanding pain, and 24 were offered a short version of the rehabilitation program for SED (12 or 16 weeks) based on a clinical assessment that the 24-week MMI was too comprehensive in these cases (Fig. 1).

All SED-patients included in the 24-week MMI at the two units were asked to participate in the study. Of the 463 patients included in the 24-week treatment, 70 declined participation. Before treatment-start, three of the patients that had initially agreed to participate terminated their treatment and were excluded. A total of 390 patients were included in the study. Eleven of these dropped out during the treatment. Consequently, 379 patients completed the 24-week MMI.

\section{Inclusion and exclusion criteria}

Inclusion criteria for the study were as follows: 1) referred for SED, fulfilled the criteria for SED; scored $>4.5$ on the Shirom-Melamed Burnout Questionnaire (SMBQ; see section measurements for more information) together with a severity assessment: The patient described a substantial functional impairment due to symptoms of SED, severely limiting the ability to participate in both work and leisure time activities previously accustomed to before the onset of SED; 2)18-65 years of age; 3 ) the patient was considered to be suitable for group treatment and logistically able to participate in treatment; 4) no known abuse of alcohol or drugs; 5) did not participate in any other form of MMI. Psychiatric comorbidity was not an exclusion criterion per se, except severe depression, moderate/high risk of suicide, psychosis, or untreated PTSD. 6) Patients who reported a severe comorbid psychiatric or somatic illness deemed to be in more acute need of treatment than SED were also excluded. Since this was an open clinical study, no specific restrictions on medications were endorsed. For baseline characteristics, see Table 1.

\section{Treatment}

After inclusion, the patients received a schedule of all appointments of the standardized team-based MMI, spanning over 24 weeks. Each multi-professional team consisted of one licensed psychologist, one licensed M.D., one licensed physiotherapist, and one rehabilitation coordinator (occupational therapist, licensed psychologist, or licensed nurse) responsible for overseeing 


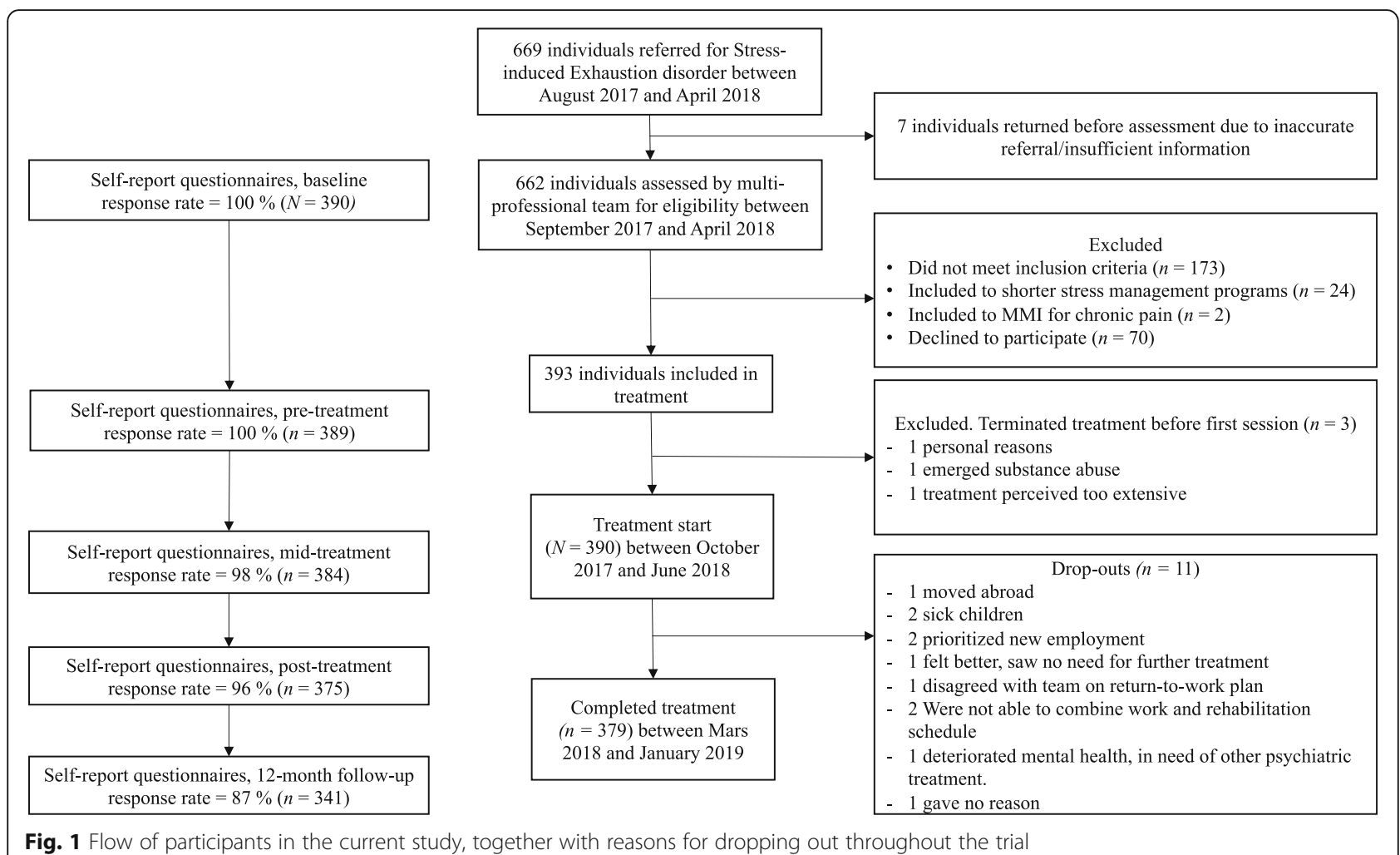

return-to-work-strategies. Although the content of the rehabilitation program and the order of the group treatments were standardized, the timing of the individual sessions, team meetings, and rehabilitation meetings could vary somewhat. For an overview of the treatment components, see Fig. 2. A more extensive outline of each component, together with the contents of each group treatment session, is supplied in the online supplement.

Legend: Overview of the 24-week Multimodal intervention for Stress-induced Exhaustion.

\section{Measurements}

Baseline characteristics and measures of psychological variables were collected during the assessment phase, at the start of rehabilitation, mid-treatment, posttreatment, and at 12-month follow-up. All questionnaires were administered digitally through a secure online-login, which is a reliable way of collecting psychological self-report measures [41]. To decrease the risk of instrumentation-bias, the order of all self-rating questionnaires was randomized at each instance of administration.

\section{Primary outcomes}

SED symptoms were measured by the Karolinska Exhaustion Disorder Scale (KEDS), which is explicitly constructed for measuring symptoms of SED [34]. KEDS consists of nine items rated on a 7-point Likert scale ranging from zero to six. The items correspond to the diagnostic criteria of SED and are formulated in line with autobiographical descriptions from patients suffering from SED. At scores $0,2,4$, and 6 on each item, different definitions are presented underneath. A score of 19 or above indicates "at risk of SED." KEDS has demonstrated good internal consistency with a reported Cronbach's alpha of .94 and has been shown to discriminate between SED-patients and healthy controls effectively. Furthermore, it is sensitive to measure changes during treatment (ibid.). Cronbach's alpha for KEDS in this study was .75 .

Working time was evaluated through the self-rated question: "How much are you working right now?" answered in percent. Only participants with some form of occupation (employed or studying; $n=365$ ) were included in the return-to-work calculations. Additionally, the self-rated question "How much compensation are you receiving from the Swedish Insurance Agency?" was administered to evaluate sick-leave compensation, answered in percent. Data from the Swedish Social Insurance Agency is to be gathered in the future and will be published elsewhere.

\section{Secondary outcomes}

Shirom-Melamed Burnout Questionnaire (SMBQ) was used to measure burnout [42]. SMBQ consists of 22 items rated on a 7-point Likert scale varying from one 
Table 1 Baseline characteristics of patients with Stress-induced Exhaustion disorder $(N=390)$ participating in a 24-week Multimodal intervention

\begin{tabular}{|c|c|c|}
\hline Characteristics & Mean & SD \\
\hline \multirow[t]{2}{*}{ Age } & 43.69 & 9.42 \\
\hline & $n$ & $\%$ \\
\hline \multicolumn{3}{|l|}{ Sex } \\
\hline - Female & 344 & 88 \\
\hline - Male & 46 & 12 \\
\hline \multicolumn{3}{|l|}{ Marital status } \\
\hline - Single or other & 131 & 33 \\
\hline - Married/living together & 238 & 61 \\
\hline - Partner (living apart) & 21 & 5 \\
\hline \multicolumn{3}{|l|}{ Education } \\
\hline - Elementary school and/or secondary school & 92 & 23 \\
\hline - University $<3$ years & 62 & 16 \\
\hline - University $\geq 3$ years & 191 & 49 \\
\hline - Other & 45 & 12 \\
\hline
\end{tabular}

Nationality

- Sweden

- European

- Other

Approved sick-leave compensation

- $0 \%$

$-25 \%$

- $50 \%$

- $75 \%$

$-100 \%$

Working time (including studies)

- 0\%

$-1-25 \%$

- 26-50\%

$-51-75 \%$

- 76-100\%

Occupational status

- Employed/self-employed

- Studying

- Unemployed

Symptom-duration before seeking treatment

- 1-12 months

- > 12 months

Previosly on sick-leave due to Stress-induced Exhaustion disorder

Comorbidity

- Suffers from some kind of physical pain

- Describes longstanding pain

- Number of patients with only Stress-induced Exhaustion disorder

- Psychiatric comorbidity 
Table 1 Baseline characteristics of patients with Stress-induced Exhaustion disorder $(N=390)$ participating in a 24-week Multimodal intervention (Continued)

\begin{tabular}{lll}
\hline Characteristics & Mean & SD \\
\hline - Somatic comorbidity & 27 & 7 \\
- Number of patients with more than one comorbid diagnosis & 32 & 8 \\
- Number of patients with both somatic and psychiatric comorbidity & 15 & 4 \\
Medications & 103 \\
- Psycholeptic sleep medication & 158 \\
- Psychoanaleptic antidepressant medication & 72 \\
- Psycholeptic sedative medication & 41 \\
- Other, incl. Pain medication, paracetamol/NSAID and medications not prescribed by a physician & 254 \\
\hline
\end{tabular}

(almost never) to seven (almost always). The scale consists of four subscales (physical fatigue, cognitive weariness, tension, and listlessness), and an overall index is calculated from the mean of all items. The SMBQ was initially developed to be used in working populations where a score of $\geq 3.75$ has been suggested as indicative of a high degree of burnout [43]. Later the SMBQ has been validated as a measure of burnout in clinical populations, suggesting a 4.4 cut-off for SED [44]. To ensure the severity of patients included within this health care imitative mentioned above (see Study design), the Health Care Services Stockholm County increased this score to 4.5. Therefore 4.5 was used as a cut-off in the current study. Cronbach's alpha for SMBQ in this study was .87 .

The Hospital Anxiety and Depression Scale (HADS) was used to measure symptoms of anxiety and symptoms of depression [45]. HADS comprises of two sets of 7-item sub-scales rated on a 4-point Likert scale ranging from zero to three (with different statements for each item), measuring anxiety and depression. Each subscale has a total score of 21 , where a score of $\geq 11$ indicates a probable caseness of a mood or anxiety disorder. In a review of 47 articles, HADS has been shown to have satisfactory internal consistency with a mean Cronbach's alpha of 0.83 for HADS-A and 0.82 for HADS-D, and to be a valid measure of change over time in depression and anxiety in relation to numerous treatments [46]. The Cronbach's alpha in this study was .79 for the anxiety scale and .78 for the depression scale.

Insomnia Severity Index (ISI) was used to assess problems with insomnia since sleep has been shown to be a predictor of return-to-work in patients receiving treatment for SED [47, 48]. ISI is a widely used measure in the research of psychological treatment for sleeping

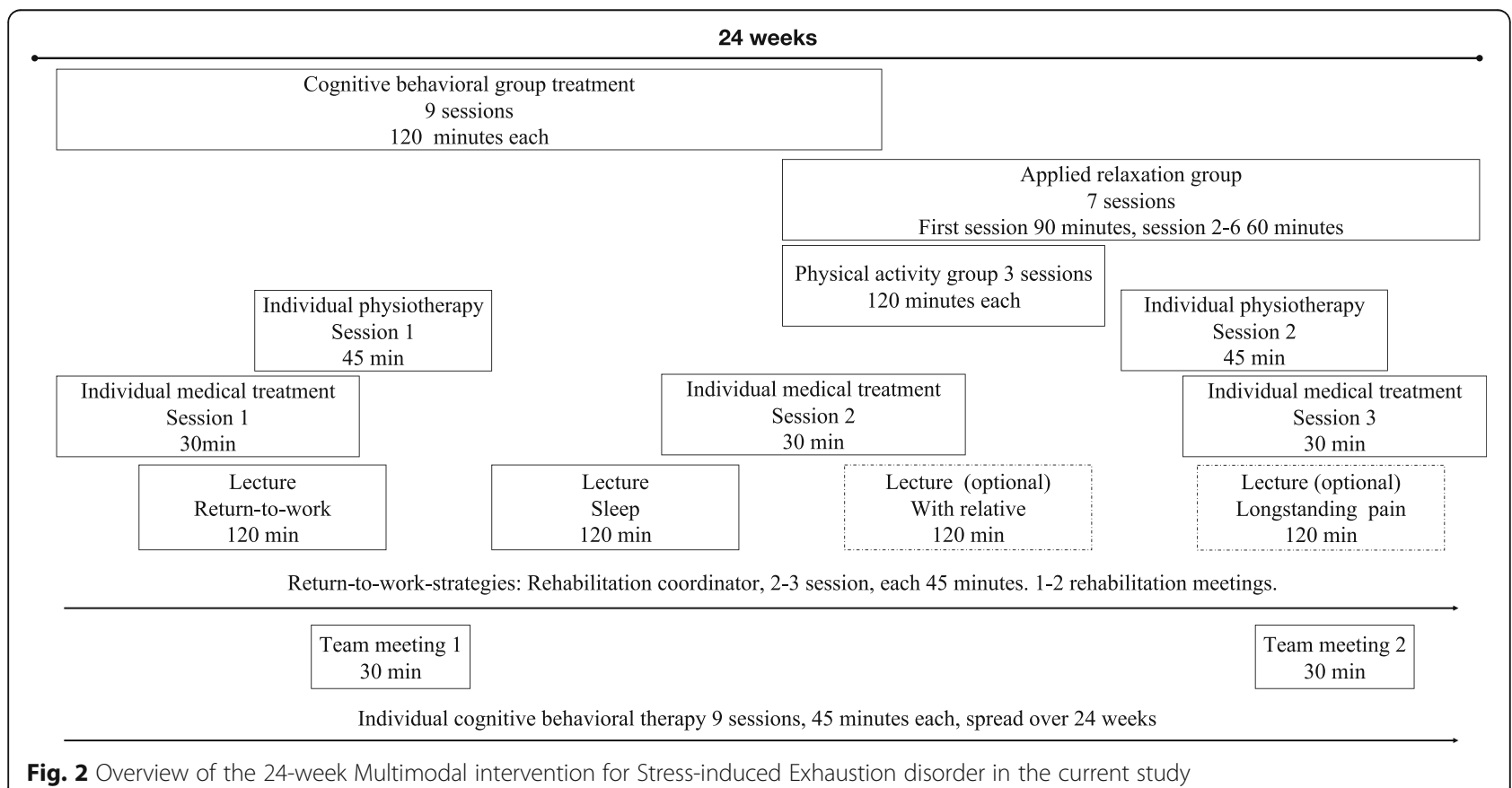

Fig. 2 Overview of the 24-week Multimodal intervention for Stress-induced Exhaustion disorder in the current study 
disorders. It has demonstrated adequate internal consistency and proven to be a reliable measure of changes in sleep during treatment [47]. Cronbach's alpha for ISI in this study was .84 .

EuroQol five dimension scale (EQ-5D) was used to measure health-related quality of life [49]. The scale consists of five questions (mobility, self-care, usual activities, pain/discomfort, and anxiety/depression), each rated on a severity level of three $(1=$ no problems, $2=$ some problems, $3=$ severe problems). The answers are measured against an index tariff based on a sample from the population. Here, the standard procedure of using the UK EQ-5D index tariff was facilitated to obtain values for the health states [50].

\section{Negative effects}

The Negative Effects Questionnaire (NEQ) was used to measure adverse events of treatment. It consists of 32 items rated on a 5-point Likert scale ranging from "not at all" to "extremely so," with a factor structure of six: symptoms, quality, dependency, stigma, hopelessness, and failure [39]. In conjunction with each item, the patient also decides whether or not he/she has experienced the adverse event at all, and whether it could be attributed to the treatment or other external circumstances. The scale has been shown to have good reliability and to effectively measure the adverse effects of psychological interventions [51]. While the NEQ is developed to measure adverse events in psychological treatments, the current treatment was multimodal. Since the MMI mainly consisted of psychological interventions, the NEQ was still deemed as a valid measure of adverse events.

\section{Treatment credibility}

The Treatment Credibility Questionnaire (TCQ) was administered at the start of treatment and mid-treatment (10 weeks after treatment start) to assess the credibility of the treatment. The TCQ has demonstrated high internal consistency and good test-retest reliability [52].

\section{Statistics}

Statistical analysis was performed in IBM SPSS Statistic version 25 (IBM Inc., Armonk, NY, USA). All repeated symptom outcome measures were analyzed using mixed-effects models, with time as a fixed effect and random intercepts. Model fit was evaluated using -2 restricted log-likelihood. Data were analyzed using an intention-to-treat procedure, meaning all patients that started treatment were included in the analysis, irrespective of completion. Missing data were handled using maximum likelihood estimation. Pearson's $x^{2}$-tests and independent samples t-tests were used to analyze potential differences between completers and drop-outs.
We calculated within-group effect sizes over time using Cohen's $d$, where $0.2-0.5$ was considered small, $0.5-0.8$ moderate, and $\geq 0.8$ large [53]. The guidelines by Jacobson and Truax [54] were utilized to evaluate clinically significant change. To meet the criteria for clinically significant change on KEDS, the participants had to demonstrate a reliable change of 8.72 and meet the criteria for clinically significant improvement. The cut off of 19 on KEDS was chosen, as this indicates "at risk of SED," based on a sample of 117 healthy individuals [34]. There are, however, currently no established criteria for clinically significant improvement in the treatment of SED, and the score of 19 has been questioned and deemed as too low for diagnostic purposes in clinical settings [20]. Because of this, complimentary analysis of clinically significant change was also performed on SMBQ to give a more balanced view of the observed improvements. On SMBQ, participants had to demonstrate a reliable change of 0.69 and score under the cut off of 4.4, based on the recommendations of Lundgren-Nilsson, Jonsdottir, Pallant \& Ahlborg [44]. Patients who did not complete post-treatment measures, as well as 12 months follow-up, were categorized as not clinically significantly improved. Furthermore, patients who reported an increase of 8.72 on KEDS at the end of treatment were categorized as deteriorated.

\section{Results}

\section{Adherence and attrition}

Of the 390 patients included, 3\% $(n=11)$ dropped out during the rehabilitation period (Fig. 1). There was a significantly lower level of education in the drop-out group $\left(x^{2}(4)=9.59, p<.05\right)$ compared to completers. There was also a significantly higher degree of insomnia measured with ISI in the population that dropped out $(M=20.09$, $S D=5.58)$ compared to the patients who completed the treatment $(M=16.27, S D=5.85) ; t(388)=2.14, p<.05$. Full characteristics for drop-outs compared with completers are presented in the online supplement.

On average the length of MMR was 23.99 weeks (SD \pm $2.85)$. Average number of completed sessions were as following: Cognitive-behavioral group treatment 7.91 $(\mathrm{SD} \pm 1.23)$ of 9; Applied relaxation group 5.36 ( $\mathrm{SD} \pm$ 1.81) of 7; Physical activity group 1.97 ( $\mathrm{SD} \pm 0.99$ ) of 3 ; Individual $\mathrm{CBT} 9.73(\mathrm{SD} \pm 0.72)$ of 10 (including 1 booster session); Individual M.D. 3.41 (SD \pm 0.72 ) of 3 ; Individual Physiotherapist $1.95(\mathrm{SD} \pm 0.76)$ of 2 ; Individual rehabilitation coordinator $1.48(\mathrm{SD} \pm 0.95)$ of $2-3$, Team meetings $1.97(\mathrm{SD} \pm 0.36)$ of 2 ; Rehabilitation meetings 1.44 ( $\mathrm{SD} \pm 1.03)$ of $1-2$; Sleep lecture 0.92 $(\mathrm{SD} \pm 0.28)$ of 1 ; Lecture on return-to-work-process 0.83 $(\mathrm{SD} \pm 0.38)$ of 1 ; Longstanding pain lecture 0.37 ( $\mathrm{SD} \pm$ 0.48 ) of 1 (optional); Lecture with relative 0.33 ( $\mathrm{SD} \pm$ 0.48 ) of 1 (optional). 


\section{Primary outcome measures}

There was a significant improvement over time on KEDS, $F(2,360.94)=445.04, p<.01$ with a large withingroup effect size at post-treatment $(d=1.61)$, which was maintained at 12-month follow-up. Means, standard deviations, and effect sizes are presented in Table 2. Figure 3 shows the change in KEDS over the course of treatment.

At post-treatment, there was an increase in average working time with $26 \%$ (where $100 \%$ is full timeemployment) in patients with some form of occupation (employed or studying; $n=365$ ), as well as a decrease in sick-leave compensation with $23 \%$ (where $100 \%$ is fulltime sick-leave compensation) in the sample as a whole $(N=390)$. At 12 -month follow-up there was a $50 \%$ increase in average working time in patients with some form of occupation (employed or studying $n=365$ ) and a $49 \%$ decrease in sick-leave compensation in the sample as a whole $(N=390)$. Means and standard deviations are presented in Table 3. To illustrate: If a participant worked $25 \%$ at the start of treatment and received $75 \%$ sick-leave compensation, at 12-month follow-up, this participant could be expected to work $75 \%(25 \%+50 \%)$ and received a sick-leave compensation of 26\% (75 $49 \%)$. A more extensive overview of the changes in the distribution of working time and sick-leave compensation is presented in the online supplement.

\section{Secondary outcome measures}

There was a significant improvement over time on HADS depression $F(2,356.00)=297.98, p<.01$, HADS anxiety $F(2,366.50)=255.04, p<.01$, SMBQ, $F(2$, $354.81)=551.18, \quad p<.01, \quad$ ISI $F(2,365.22)=254.44$, $p<.01$ and EQ. 5D $F(2,366.67)=151.80, p<.01$, with overall large within-group effects sizes at post-treatment $(d=0.91-1.75)$. All these improvements were maintained at 12-month follow-up. Means, standard deviation, and effect sizes are presented in Table 2. Figure 4 shows changes in mean scores over time on HADS for both subscales of depression and anxiety.

\section{Clinical significant change}

At the end of treatment, the proportion of patients fulfilling the criteria for clinically significant change on KEDS was $27 \%(n=106)$. At 12 -month follow-up, this change was increased to $37 \%(n=143)$. On SMBQ, the proportion of patients fulfilling the criteria for clinically significant change was $57 \%(n=222)$, which was maintained at $57 \%(n=223)$ at 12 -month follow-up.

\section{Negative effects}

In total, $57 \%(n=224)$ reported some form of adverse effect attributed to treatment, mainly concerning an increase in stress, anxiety, and worry. The most frequently reported question was, "I felt like I was under more stress" at $26 \%(n=97)$. No participant reported an increase of 8.72 of their KEDS score post-treatment compared to their baseline score, showing a deterioration rate of $0 \%(n=0)$.

\section{Discussion}

This open clinical trial explored changes in symptoms and return-to-work-rates in 390 SED-patients participating in a standardized MMI in a clinical setting. It also evaluated the negative effects of said treatment. In general, there were significant and large improvements in all measures. Patients showed reduced symptoms of SED, burnout, insomnia as well as anxiety and depression following treatment, with large within-group effect sizes at 12-month follow-up $(d=0.91-1.76)$. Patients also

Table 2 Means, return-to-work-rates and within-group effects sizes (Cohen's d) of symptoms over time in patients with Stressinduced Exhaustion disorder ( $N=390)$ participating in a 24-week Multimodal intervention

\begin{tabular}{|c|c|c|c|c|c|}
\hline \multirow[t]{2}{*}{ Measure } & \multicolumn{3}{|l|}{ Mean (SD) } & \multicolumn{2}{|c|}{ Within-group effect size } \\
\hline & Pre & Post & $12 \mathrm{MFU}$ & pre-post & pre-12MFU \\
\hline KEDS & $34.88(6.18)$ & $23.35(8.09)$ & $21.84(9.13)$ & 1.61 & 1.69 \\
\hline Working time* \% & $26(34.4)$ & $52(29.92)$ & $76(34.31)$ & & \\
\hline SLC \% & $62(41.7)$ & $39(31.7)$ & $12(29.9)$ & & \\
\hline \multicolumn{6}{|l|}{ HADS } \\
\hline - anxiety & $11.25(4.07)$ & $6.92(3.64)$ & $6.71(3.86)$ & 1.12 & 1.15 \\
\hline - depression & $11.22(3.84)$ & $6.50(3.92)$ & $6.26(4.30)$ & 1.22 & 1.23 \\
\hline SMBQ & $5.63(0.63)$ & $4.00(1.11)$ & $3.87(1.24)$ & 1.75 & 1.76 \\
\hline$|S|$ & $16.38(5.87)$ & $9.92(5.75)$ & $9.81(6.06)$ & 1.11 & 1.10 \\
\hline EQ 5D & $0.61(0.16)$ & $0.75(0.14)$ & $0.76(0.17)$ & 0.91 & 0.92 \\
\hline
\end{tabular}

Pre before treatment; Post after treatment; 12MFU 12-month follow-up; KEDS Karolinska Exhaustion Disorder Scale; SLC Sick-leave compensation; SMBQ ShiromMelamed Burnout Questionnaire; HADS Hospital Anxiety and Depression Scale; ISI Insomnia Severity Index; EQ-5D EuroQol five dimension scale. *Patients with some form of occupation, employed or studying $(n=365)$ 


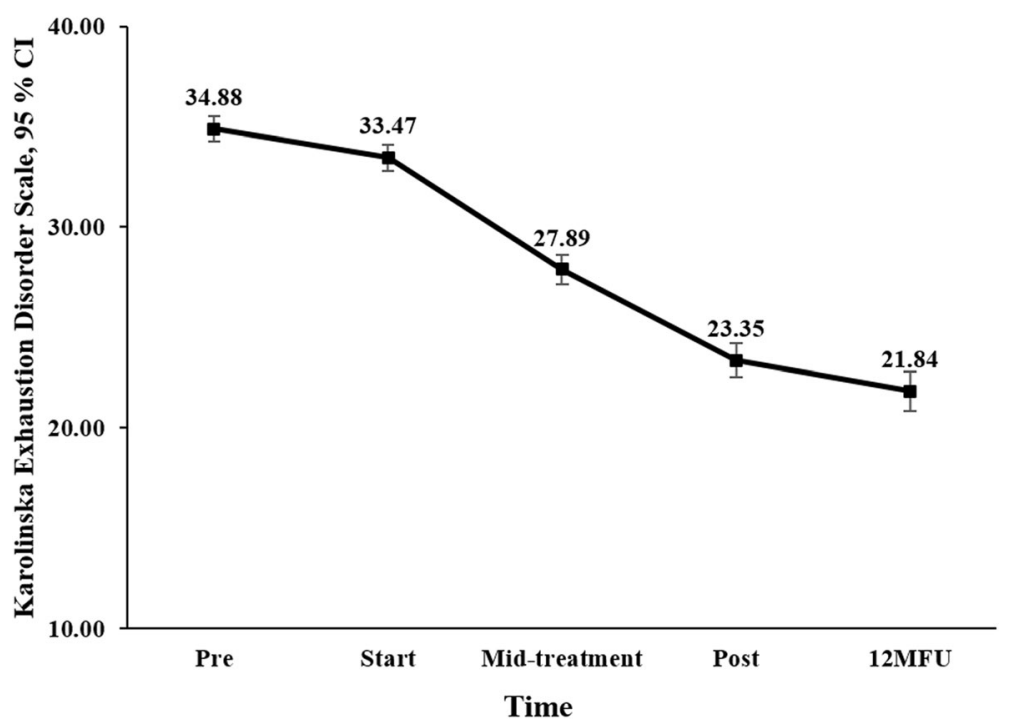

Fig. 3 Changes in mean scores (intention-to-treat procedure) on Karolinska Exhaustion Disorder Scale in patients with Stress-induced Exhaustion disorder $(N=390)$ participating in a 24-week Multimodal intervention. Pre, before treatment; Start, treatment start; Mid-treatment, 12 weeks into treatment; Post, after treatment; 12MFU, 12-month follow-up

reported a significant increase in quality of life. At 12month follow-up, 37\% $(n=143)$ of the patients achieved clinically significant change on KEDS and $57 \%(n=223)$ measured by SMBQ. Beyond these improvements, patients with some form of occupation (employed or studying; $n=365$ ) had returned to work with an average of $50 \%$, and in the sample as a whole $(N=390)$ sickleave compensation was reduced by $49 \%$ at 12 -month follow-up. While a few patients reported adverse effects of treatment, mainly concerning an increase in symptoms of stress, the overall impression is that SEDpatients participating in a standardized MMI reported symptom alleviation, an increase in working time, a decrease in sick-leave compensation, and enhanced quality of life.

\section{Changes in symptoms}

The reduction in burnout was comparable to or larger than with previous studies $[24,27,28]$. While no causal inferences can be made, these improvements are



Fig. 4 Changes in mean scores (intention-to-treat procedure) on the subscales depression and anxiety of the Hospital Anxiety and Depression Scale, in patients with Stress-induced Exhaustion disorder $(N=390)$ participating in a 24-week Multimodal intervention. Pre, before treatment; Start, treatment start; Mid-treatment, 12 weeks into treatment; Post, after treatment; 12MFU, 12-month follow-up 
promising given that the MMI extends over 12 months in Stenlund et al. [28], and 18 months in Glise et al. [24], compared to 6 months in the current study.

Continuous measurements of burnout, anxiety, and depression have been administered to a large SEDpopulation once before. In Glise et al. [24], a marked decrease in anxiety and depression (measured with HADS) was observed after 3 months of treatment, after which levels continued to decrease further to subclinical levels. Meanwhile, symptoms of burnout steadily declined, yet remained above sub-clinical levels at an 18-month follow-up. In the current study, the same pattern was recognized. Here, SED-patients reported a high degree of comorbid symptoms of anxiety and depression at the start of treatment. These symptoms successively decreased as treatment was initiated, while symptoms of burnout (and in this case also SED) declined, but still persisted to a higher degree at 12-month follow-up (21.8 on KEDS and 3.87 on SMBQ). Because a score of $\geq 19$ on KEDS is indicative of SED, and a score of $\geq 3.75$ is indicative of a "high degree of burnout" [34, 43], the symptoms of SED that remain are, despite being substantially improved, still above sub-clinical levels.

Patients in the current study reported a decrease in insomnia with a large effects-size, which was maintained at the 12-month follow-up. This decrease is encouraging, given that improved sleep quality has been shown to play a vital role in the effective treatment of SED $[6,48,55]$.

\section{Return to work}

Concerning return-to-work rates, one previous study of MMI for SED has reported return-to-work-numbers before and after treatment. In the study by Stenlund, Nordin, and Järvholm [56], participants reported an average of $72 \%$ on full-time sick-leave at baseline, $60 \%$ at the end of treatment (12 months later), and $38 \%$ at 12 month follow-up (personal communication with the author).

The sick-leave rates in the current study are markedly lower than in Stenlund, Nordin, and Järvholm [56]. These differences could, of course, mirror differences in the administration of sick-leave insurance, which is affected by factors such as changes in political policies over time and varying regional bureaucratic traditions. They could also be a reflection of dissimilarities in the number of sick days before starting treatment in the above-mentioned populations. Because of this, comparisons with the current study, of course, can not tell us anything about which of these interventions is more effective. What it can tell us is that the positive returnto-work-rates in the current study may be interpreted as an indication of an effective return-to-work-intervention.

\section{Negative effects}

To our knowledge, this is the first trial where the NEQ has been used in face-to-face clinical treatment, and the adverse effects do not appear to stand out. Compared to large samples of patients receiving CBT administered via the internet and a survey among respondents from a psychiatric population receiving psychological treatment, the adverse effects are comparable to or lower than in the current study [57]. Also, rates of deterioration are lower compared to the general psychiatric and primary care populations in Sweden [58, 59]. These finding, together with the relatively low rate of drop-outs (3\%), implies that this MMI does not seem to pose a higher risk of harm than other unimodal psychological treatment strategies.

\section{Limitations and strengths}

There are several strengths and weaknesses in the current study that need to be highlighted. First and foremost, this open clinical trial is not controlled, which impedes any causal inferences between observed changes and the treatment administered. However, the duration of symptoms of SED before starting treatment varied across participants, which - together with the comparisons of SMBQ in studies made previously - indicate that the observed changes are probably not exclusively the result of the natural course of SED, spontaneous improvement, or regression to the mean.

Even though the treatment was standardized, there are still some parts of the treatment that were not (the content of the individual sessions). This limitation reduces the internal and external validity of this study. Another limitation is the exclusive use of self-report measures. An improvement would have been to use another form of outcome procedures, such as standardized clinician ratings. It would, of course, also have been more reliable to evaluate return-to-work through other measures than self-rating-questionnaires. One option is to retrieve actual sick-leave data from the Swedish Social Insurance Agency, which will be carried out in a later publication.

As far as we know, this population is the largest population of SED-patients to date, to have been tracked through an intervention, ensuring a high power and clinically representative population. All patients were medically examined before treatment and were diagnosed by a team of professionals, including a licensed physician and a licensed psychologist. Thanks to this procedure, comorbid diagnosis, and medications, including Anatomical Therapeutic Chemical Classification (ATC-codes), are accounted for. Furthermore, the order of all questionnaires was randomized at each instance of administration to reduce the risk of instrumentation bias. Another strength is the use of a standardized treatment with thoroughly described treatment content. Even 
though we cannot infer any change from treatment, we can at least conclude that overall, all patients received the same treatment, except for some content in the individual sessions.

The fact that the study was set at two different locations in Stockholm and had a large number of clinicians delivering the treatment increases the generalizability of the study and significantly increases the natural and external validity, as well as decreases the risk of therapist-bias.

Despite being set in a naturalistic clinical context, the drop-out rate is low, and the response rate is relatively high for this setting. This further increases generalizability of the data to other settings. It can be assumed that the patients, overall, perceived the intervention as relevant. Lastly, there is a low exclusion rate in the recruitment phase, ensuring that the sample treated in the current study can be considered clinically representative of an actual SED-population.

\section{Future research}

As far as we know, most existing published trials compare variations of the same MMI, and no study has yet compared MMI to a wait-list-control. Therefore, there is an apparent need for randomized controlled trials to ascertain the efficacy of MMI for SED. Beyond this, there is a need to differentiate what components of MMI lead to symptom-improvement. MMI is the recommended modality of treatment for SED, even though the evidence is scarce. It would be interesting to compare a full MMI to a less cumbersome, unimodal treatment, such as an individual CBT or individual physiotherapy with physical exercise, or just return-to-work-strategies. Since the most substantial symptom improvement shown to date in SED-patients resulted from an internet-delivered treatment [60], looking at other modes of treatment besides MMI should be encouraged. This is especially true considering the administration of MMI can be logistically cumbersome and resource-demanding, with the risk of decreasing access to treatment.

Other treatment protocols of common mental disorders (CMD), such as behavioral activation for depression, or Clark's treatment for social anxiety, rest upon a theoretically founded framework of how the condition arises and is maintained [61, 62]. An equivalent theoretical framework for the cause and maintenance of SED is currently missing in the clinical treatment literature. Therefore, it is relevant to explore potential predictors and process variables to increase understanding of the theoretical conceptualization and treatment of SED.

A few observations about the sample are also worth mentioning: $40 \%(n=155)$ are diagnosed with other psychiatric diagnosis, $41 \%(n=158)$ are on antidepressant medications, and $66 \%(n=257)$ describe some form of comorbid pain. Furthermore, 35\% $(n=137)$ of the patients reported previously being on sick leave due to SED. In line with previous studies [24, 25], these observations highlight a considerable burden of disease regarding both mental and somatic symptoms in SEDpatients, which need to be accounted for in clinical care and further explored in future research.

There was a considerable discrepancy in the number of participants achieving clinically significant change when measured by KEDS compared to SMBQ. This indicates that SMBQ and KEDS are not mutually overlapping questionnaires, and probably do measure different underlying constructs, in accordance with previous research [33]. Because of this, the differences between SMBQ and KEDS should to be explored more thoroughly in future research.

One last thing to note is that regardless of how clinically significant change was calculated, a large proportion of the population did not reach a clinically significant change at 12-months follow up (63\% based on KEDS, $43 \%$ based on SMBQ). This, together with the relative increase in variation in the KEDS measurements at post- and 12-month follow-up, compared to the premeasurements, suggest that different patients fare differently in treatment. Little is still known about the population with SED. Therefore, future research should explore the possibilities of sub-groups through cluster analysis.

\section{Conclusions}

SED-patients participating in this standardized MMI reported large symptom alleviation, increased working time, and reduced sick-leave compensation, suggesting that they did seem to benefit from treatment. While no causal inferences can be made, comparisons with previously published treatment studies indicate that observed improvements, in part, are attributable to treatment. Furthermore, this study confirms previous findings that high levels of depression and anxiety decrease to subclinical levels during treatment, while symptoms of SED also decline, yet still persists above sub-clinical levels at 12-month follow-up. There were some negative effects, but no more so than other psychological treatments. In conclusion, findings suggest that a standardized MMI, administered in a clinical setting, improves symptoms and return-to-work-rates in a clinically representative SED-population.

\section{Supplementary information}

Supplementary information accompanies this paper at https://doi.org/10. 1186/s12888-020-02907-3.

Additional file 1. Treatment overview supplement.

Additional file 2. Extended demography supplement.

Additional file 3. Supplementary figures of return-to-work-rates. 


\section{Abbreviations}

CBT: Cognitive Behavioral Therapy; ISI: Insomnia Severity Index; KEDS: Karolinska Exhaustion Disorder Scale; MMI: Multimodal Intervention; SMBQ: Shirom-Melamed Burnout Questionnaire; SED: Stress-induced Exhaustion disorder; HADS: The Hospital Anxiety and Depression Scale; TCQ: The Treatment Credibility Questionnaire

\section{Acknowledgments}

Not applicable.

\section{Authors' contributions}

$J C v d L, G B J, A R$ and $M B$ were all contributors to the design and planning of the current study. JCvdL and GBJ oversaw the implementation of the study procedure at the clinical health care-centers, and JCvdL was responsible for the data collection. MB was responsible for clinical teamcounseling at both health-care centers promoting uniformity in treatment adherence among all MMl-teams. GBJ was responsible for the identification and categorization of diagnoses and medications. AR was responsible for all implementation and analysis of the adverse effects. $J C v d L, F \AA$, and $M B$ were all responsible for data analysis. JCvdL wrote the bulk of the manuscript, and $F \AA, G B J$, and $M B$ were all major contributors to the writing of the manuscript. All authors read and approved the final manuscript."

\section{Funding}

This study was funded by a research grant (nr 2018-00154) from "FORTE: Swedish Research Council for Health, Working Life and Welfare." FORTE had no influence in the design of the study, data collection, analysis, and interpretation of data and in writing the manuscript. Open access funding provided by Uppsala University.

\section{Availability of data and materials}

The datasets generated and analyzed during the current study are not publicly available because this data is part of a larger data-collection that is currently ongoing. The data is available from the corresponding author on a reasonable request.

\section{Ethics approval and consent to participate}

This study was registered on Clinicaltrials.gov (Identifier: NCT03360136), approved by the Regional Ethical Review Board in Stockholm, Sweden (Approval Nr. 2016/1834-31/2), and followed the ethical principles of the Declaration of Helsinki.

At inclusion, verbal and written information about the study was administered. The patients were informed that participating in the study was voluntary, that they could terminate their participation at any point, and that their participation would in no way affect the contents of their rehabilitation. All participants provided written consent before inclusion.

\section{Consent for publication}

$$
\text { Not applicable. }
$$

\section{Competing interests}

The authors declare that they have no competing interests.

\section{Author details}

1Department of Psychology, Uppsala University, Von Kraemers allé 1A, SE-752 37 Uppsala, Sweden. ${ }^{2}$ Department of Psychology and Social Work, Mid Sweden University, Kunskapens väg 1, SE- 83140 Östersund, Sweden. ${ }^{3}$ Department of Clinical Neuroscience, Karolinska Institute, Tomtebodavägen 18A, SE-171 76 Stockholm, Sweden. ${ }^{4}$ Division of Rehabilitation Medicine, Department of Clinical Sciences, Karolinska Institute Danderyds University Hospital, SE-182 57 Stockholm, Sweden.

\section{Received: 29 April 2020 Accepted: 30 September 2020}

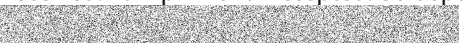

\section{References}

1. OECD. Sick on the Job? [Internet]. OECD; 2012. 212 p. (Mental Health and Work). Available from: https://www.oecd-ilibrary.org/content/publication/ 9789264124523-en.

2. Hewlett E, Moran V. Making Mental Health Count: The Social and Economic Costs of Neglecting Mental Health Care [Internet]. OECD Health Policy
Studies. OECD; 2014. 1-8 p. (Mental Health and Work). Available from: https://www.oecd-ilibrary.org/social-issues-migration-health/mental-healthand-work_9789264124523-en.

3. European Commission. EU Occupational Safety and Health (OSH) Strategic Framework 2014-2020. 2014;332. Available from: http://eur-lex.europa.eu/ legal-content/EN/TXT/PDF/?uri=CELEX:52014DC0332.

4. Eurofound. Burnout in the workplace: A review of data and policy responses in the EU [Internet]. Publications Office of the European Union. 2018. 1-41 p. Available from: http://eurofound.link/ef18047.

5. Grossi G, Perski A, Osika W, Savic I. Stress-related exhaustion disorder clinical manifestation of burnout? A review of assessment methods, sleep impairments, cognitive disturbances, and neuro-biological and physiological changes in clinical burnout. Scand J Psychol. 2015;56(6): 626-36.

6. Santoft F, Salomonsson S, Hesser H, Lindsäter E, Ljótsson B, Lekander M, et al. Mediators of change in cognitive behavior therapy for clinical burnout. Behav Ther [internet]. 2019;50(3):475-88. Available from. https://doi.org/10. 1016/j.beth.2018.08.005.

7. Schaufeli WB, Leiter MP, Maslach C. Burnout: 35 years of research and practice. Career Dev Int. 2009;14(3):204-20.

8. Bianchi $\mathrm{R}$, Schonfeld IS, Laurent E. Burnout: moving beyond the status quo. Int J Stress Manag. 2019;26(1):36-45.

9. Schonfeld IS, Bianchi R. Burnout and depression: two entities or one? J Clin Psychol. 2016;72(1):22-37.

10. Koutsimani P, Montgomery A, Georganta K. The relationship between burnout, depression, and anxiety. In: A systematic review and meta-analysis. Vol. 10, Frontiers in Psychology. Frontiers Media S.A; 2019.

11. American Psychiatric Association. Diagnostic and statistical manual of mental disorders : DSM-5. 5th ed. Washington, DC: American Psychiatric Association Arlington, VA; 2013.

12. World Health Organization. The ICD-10 classification of mental and Behavioural disorders. Geneva: World Health Organization; 1992.

13. Wallensten J, Åsberg M, Wiklander M, Nager A. Role of rehabilitation in chronic stress-induced exhaustion disorder: A narrative review [Internet]. Vol. 51, Journal of Rehabilitation Medicine. 2019. p. 331-42. Available from: https://www.medicaljournals.se/jrm/content/abstract/10.2340/16501 977-2545.

14. Höglund P, Hakelind C, Nordin S. Severity and prevalence of various types of mental ill-health in a general adult population: age and sex differences. BMC Psychiatry. 2020;20(1):1-11.

15. Krabbe D, Ellbin S, Nilsson M, Jonsdottir IH, Samuelsson H. Executive function and attention in patients with stress-related exhaustion: perceived fatigue and effect of distraction. Stress [Internet]. 2017;20(4):333-40 Available from: https://doi.org/10.1080/10253890.2017. 1336533.


metabolomic patterns in patients with exhaustion disorder. Stress [Internet]. 2019;22(1):17-26 Available from: http://www.tandfonline.com/action/ journallnformation?journalCode=ists20.

17. Savic I. MRS shows regionally increased glutamate levels among patients with exhaustion syndrome due to occupational stress. Cereb Cortex [Internet]. 2020;30(6):3759-70 Available from: https://academic.oup.com/ cercor/article/30/6/3759/5706940.

18. Dalgaard VL, Aschbacher K, Andersen JH, Glasscock DJ, Willert MV, Carstensen O, et al. Return to work after work-related stress: a randomized controlled trial of a work-focused cognitive behavioral intervention. Scand J Work Environ Health. 2017;43(5):436-46.

19. Netterstrøm B, Friebel L, Ladegaard $Y$. Effects of a multidisciplinary stress treatment programme on patient return to work rate and symptom reduction: results from a randomised, wait-list controlled trial. Psychother Psychosom [internet]. 2013;82(3):177-86 Available from: http://www.ncbi. nlm.nih.gov/pubmed/23548852.

20. Kristiansen J, Friborg MK, Eller N, Brandt LPA, Glasscock DJ, Pihl-Thingvad J, et al. Comparison of exhaustion symptoms in patients with stress-related and other psychiatric and somatic diagnoses. BMC Psychiatry [Internet]. 2019;19(1):84 Available from: https://bmcpsychiatry.biomedcentral.com/ articles/10.1186/s12888-019-2066-y.

21. Lipsitt DR. Is Today's 21st century burnout 19th Century's neurasthenia? J Nerv Ment Dis. 2019;207(9):773-7.

22. Overholser JC, Beale EE. Neurasthenia: modern malady or historical relic? Nerv Ment Dis. 2019;207(9):731-9. 
23. Heinemann LV, Heinemann T. Burnout research: Emergence and scientific investigation of a contested diagnosis. SAGE Open. 2017;7:1.

24. Glise K, Ahlborg G, Jonsdottir IH. Course of mental symptoms in patients with stress-related exhaustion: does sex or age make a difference? BMC Psychiatry. 2012;12(18):1-12.

25. Glise K, Ahlborg G Jr, Jonsdottir IH. Prevalence and course of somatic symptoms in patients with stress-related exhaustion: does sex or age matter. BMC Psychiatry. 2014;14:1-12.

26. Kamper SJ, Apeldoorn AT, Chiarotto A, Smeets RJEM, Ostelo RW, Guzman J, et al. Multidisciplinary biopsychosocial rehabilitation for chronic low back pain. Cochrane Database Syst Rev [Internet]. 2014;(9) Available from:. https:// doi.org/10.1002/14651858.CD000963.pub3.

27. Malmberg Gavelin H, Eskilsson T, Boraxbekk C-JJ, Josefsson M, Stigsdotter Neely A, Slunga Järvholm L, et al. Rehabilitation for improved cognition in patients with stress-related exhaustion disorder: RECO - a randomized clinical trial. Stress [internet]. 2018;21(4):279-91. Available from. https://doi. org/10.1080/10253890.2018.1461833.

28. Stenlund T, Ahlgren C, Lindahl B, Burell G, Steinholtz K, Edlund C, et al. Cognitively oriented behavioral rehabilitation in combination with qigong for patients on long-term sick leave because of burnout: REST-A randomized clinical trial. Int J Behav Med. 2009:16(3):294-303.

29. Salomonsson S, Hedman-Lagerlöf E, Öst L-G. Sickness absence: a systematic review and meta-analysis of psychological treatments for individuals on sick leave due to common mental disorders. Psychol Med. 2018;48(12):1954-65.

30. Salomonsson S, Santoft F, Lindsäter E, Ejeby K, Ljótsson B, Öst L-G, et al. Cognitive-behavioural therapy and return-to-work intervention for patients on sick leave due to common mental disorders: a randomised controlled trial. Occup Environ Med [Internet]. 2017;74(12):905-12 Available from: http://oem.bmj.com/content/early/2017/07/28/oemed-2 017-104342.abstract.

31. Finnes A, Ghaderi A, Dahl J, Nager A, Enebrink P. Randomized controlled trial of acceptance and commitment therapy and a workplace intervention for sickness absence due to mental disorders. J Occup Health Psychol [Internet]. 2019;24(1):198-212 Available from: http://doi.apa.org/getdoi. cfm?doi=10.1037/ocp0000097.

32. Karlson B, Jönsson P, Österberg K. Long-term stability of return to work after a workplace-oriented intervention for patients on sick leave for burnout. BMC Public Health [Internet]. 2014;14(1):821. Available from: http:// bmcpublichealth.biomedcentral.com/articles/10.1186/1471-2458-14-821.

33. Saboonchi F, Perski A, Grossi G. Validation of Karolinska exhaustion scale: psychometric properties of a measure of exhaustion syndrome. Scand J Caring Sci. 2013;27(4):1010-7.

34. Besèr A, Sorjonen K, Wahlberg K, Peterson U, Nygren Å, Åsberg M. Construction and evaluation of a self rating scale for stress-induced exhaustion disorder, the Karolinska exhaustion disorder scale. Scand J Psychol. 2014;55(1):72-82.

35. Ellbin $\mathrm{S}$, Engen $\mathrm{N}$, Jonsdottir $\mathrm{H}$, Nordlund AlK. Assessment of cognitive function in patients with stress-related exhaustion using the cognitive assessment battery (CAB). J Clin Exp Neuropsychol [Internet]. 2017;40(6):1-9 Available from: https://www.tandfonline.com/doi/full/10.1080/13803395.201 7.1388359 .

36. Jonsdottir IH, Nordlund A, Ellbin S, Ljung T, Glise K, Währborg P, et al. Cognitive impairment in patients with stress-related exhaustion. Stress 2013;16(2):181-90.

37. Boswell JF, Kraus DR, Miller SD, Lambert MJ. Implementing routine outcome monitoring in clinical practice: benefits, challenges, and solutions. Psychother Res. 2015;25(1):6-19.

38. Loerinc AG, Meuret AE, Twohig MP, Rosenfield D, Bluett EJ, Craske MG. Response rates for CBT for anxiety disorders: need for standardized criteria. Clin Psychol rev [internet]. 2015;42:72-82. Available from: http://www.ncbi. nlm.nih.gov/pubmed/26319194

39. Rozental A, Kottorp A, Boettcher J, Andersson G, Carlbring P. Negative effects of psychological treatments: an exploratory factor analysis of the negative effects questionnaire for monitoring and reporting adverse and unwanted events. PLoS One. 2016;11(6):1-22.

40. Rozental A, Boettcher J, Andersson G, Schmidt B, Carlbring P. Negative effects of internet interventions: a qualitative content analysis of patients' experiences with treatments delivered online. Cogn Behav Ther [Internet]. 2015;44(3):1-14 Available from: http://www.tandfonline.com/doi/abs/10.1 080/16506073.2015.1008033.
41. Hedman E, Ljótsson B, Rück C, Furmark T, Carlbring P, Lindefors N, et al. Internet administration of self-report measures commonly used in research on social anxiety disorder: a psychometric evaluation. Comput Human Behav [Internet]. 2010;26(4):736-40. Available from:. https://doi.org/10.1016/i. chb.2010.01.010

42. Melamed S, Kushnir T, Shirom A. Burnout and Risk Factors for Cardiovascular Diseases. Vol. 18: Behavioral medicine, Washington, D.C.; 1992. p. 53-60.

43. Grossi G, Perski A, Evengård B, Blomkvist V, Orth-Gomér K. Physiological correlates of burnout among women. J Psychosom Res. 2003;55(4):309-16.

44. Lundgren-Nilsson Å, Jonsdottir IH, Pallant J, Ahlborg G. Internal construct validity of the Shirom-Melamed Burnout Questionnaire (SMBQ). BMC Public Health [Internet]. 2012;12(1):1. Available from: http://www.biomedcentral. com/1471-2458/12/1.

45. Zigmond AS, Snaith RP. The hospital anxiety and depression scale. Acta Psychiatr Scand [internet]. 1983;67(6):361-70. Available from. https://doi.org/ 10.1111/j.1600-0447.1983.tb09716.x.

46. Bjelland I, Dahl AA, Haug TT, Neckelmann D. The validity of the hospital anxiety and depression scale. J Psychosom Res [Internet]. 2002;52:69-77 Available from: http://www.sciencedirect.com/science/article/pii/S00223 99901002963

47. Bastien $\mathrm{CH}$, Vallières $\mathrm{A}$, Morin $\mathrm{CM}$. Validation of the insomnia severity index as an outcomne measuer for insomnia research. Sleep Med. 2001;2:297-307.

48. Ekstedt M, Söderström M, Åkerstedt T. Sleep physiology in recovery from burnout. Biol Psychol. 2009;82(3):267-73.

49. The EuroQol Group. EuroQol - a new facility for the measurement of healthrelated quality of life. Health Policy (New York) [Internet]. 1990;16(3):199208. Available from: http://www.sciencedirect.com/science/article/pii/016 8851090904219.

50. Burstrom K, Johannesson M, Diderichsen F. Swedish population healthrelated quality of life results using the EQ-5D. Qual Life Res [Internet]. 2001; 10(7):621-35 Available from: http://www.ncbi.nlm.nih.gov/pubmed/ 11822795\%5Cnhttp://download.springer.com/static/pdf/975/art:10.1023/A: 1013171831202.pdf?auth66=1383404506 dcc0d49da42842b10f4758734c125b8d\&ext=.pdf.

51. Rozental A, Kottorp A, Forsström D, Månsson K, Boettcher J, Andersson G, et al. The negative effects questionnaire: psychometric properties of an instrument for assessing negative effects in psychological treatments. Behav Cogn Psychother [Internet]. 2019;47(5):559-72 Available from: https://www. cambridge.org/core/product/identifier/S1352465819000018/type/journal_ article.

52. Devilly GJ, Borkovec TD. Psychometric properties of the credibility/ expectancy questionnaire. J Behav Ther Exp Psychiatry. 2000;31(2):73-86.

53. Cohen J. Statistical power analysis for the behavioral sciences (2nd ed.). 2nd ed. Hillsdale, NJ: Lawrence Earlbaum Associates; 1988.

54. Jacobson NS, Truax P. Clinical Significance: A Statistical Approach to Denning Meaningful Change in Psychotherapy Research; 1991. p. 12-9.

55. Söderström M, Jeding K, Ekstedt M, Perski A, Åkerstedt T. Insufficient sleep predicts clinical burnout. J Occup Health Psychol. 2012;17(2):175-83.

56. Stenlund T, Nordin M, Järvholm LS. Effects of rehabilitation programmes for patients on long-term sick leave for burnout: a 3-year follow-up of the rest study. J Rehabil Med. 2012;44(8):684-90.

57. Rozental A, Magnusson K, Boettcher J, Andersson G, Carlbring P. For better or worse: an individual patient data meta-analysis of deterioration among participants receiving internet-based cognitive behavior therapy. J Consult Clin Psychol. 2017;85(2):160-77.

58. Rheker J, Beisel S, Kräling S, Rief W. Rate and predictors of negative effects of psychotherapy in psychiatric and psychosomatic inpatients. Psychiatry Res [internet]. 2017;254:143-50 Available from: http://www.ncbi.nlm.nih.gov/ pubmed/28460285.

59. Mechler J, Holmqvist R. Deteriorated and unchanged patients in psychological treatment in Swedish primary care and psychiatry. Nord J Psychiatry [internet]. 2016;70(1):16-23. Available from. https://doi.org/10. 3109/08039488.2015.1028438.

60. Lindsäter E, Axelsson E, Salomonsson S, Santoft F, Ejeby K, Ljótsson B, et al. Internet-based cognitive behavioral therapy for chronic stress: a randomized controlled trial. Psychother Psychosom [Internet]. 2018;87(5): 296-305 Available from: https://www.karger.com/Article/FullText/490742.

61. Clark DM, Ehlers A, McManus F, Hackmann A, Fennell M, Campbell H, et al. Cognitive therapy versus fluoxetine in generalized social phobia: a randomized placebo-controlled trial. J Consult Clin Psychol [Internet]. 
2003;71(6):1058-67 Available from: http://www.ncbi.nlm.nih.gov/ pubmed/14622081.

62. Martell CR, Dimidjian S, Herman-Dunn R. Behavioral activation for depression: A clinician's guide. Behavioral activation for depression: A clinician's guide. New York, NY: Guilford Press; 2010. xvi, 220-xvi, 220.

\section{Publisher's Note}

Springer Nature remains neutral with regard to jurisdictional claims in published maps and institutional affiliations.

Ready to submit your research? Choose BMC and benefit from:

- fast, convenient online submission

- thorough peer review by experienced researchers in your field

- rapid publication on acceptance

- support for research data, including large and complex data types

- gold Open Access which fosters wider collaboration and increased citations

- maximum visibility for your research: over $100 \mathrm{M}$ website views per year

At $B M C$, research is always in progress.

Learn more biomedcentral.com/submissions 